\title{
La evaluación como instrumento de aprendizaje en programación de computadores usando Conectivismo y brain based learning
}

\author{
Assessment as a Learning instrument in a computer programming course using \\ connecctivism and BBL
}

\author{
O. I. Trejos-Buriticá iD; L. E. Muñoz-Guerrero iD; G. R. Solarte-Martínez iD
}

\begin{abstract}
This article presents results, analysis and discussion derived from a research made in a Computer Programming course in Systems and Computer Engineering whose objective was to establish comparative criteria that would capitalize the advantages of Connectivism with windows of time, as a learning instrument, in comparison with methodology used normally in class especially in evaluation by individual written exams. We proceeded to work forming four subgroups, from ten subgroups, so each one responds to a different methodology of evaluation to compare them quantitatively and qualitatively. The results show great favorability towards the evaluation methodology supported in Conectivismo. It is concluded that, in a controlled environment, taking advantage of the options provided by the Connectivism, it is possible to achieve the motivation required for a more effective, more lasting learning and more tuned to the natural language of today's young people: technology.
\end{abstract}

Index Terms —Assessment, Computing, Connectivism, Learning, Programming

Resumen - Este artículo compara la metodología tradicional de enseñanza de la programación frente a la teoría moderna del Conectivismo y su complemento con el aprendizaje basado en el cerebro (BBL) en un curso de programación de computadores de Ingeniería de Sistemas. A partir de los resultados cualito cuantitativos comparativos de evaluaciones escritas se estableció su impacto como instrumento de aprendizaje. Se procedió a trabajar con 16 grupos en paralelo, de $\mathbf{4 0}$ grupos en total en los semestres académicos del 2015 al 2018, de forma que se aplicara una metodología diferente de evaluación para compararlas a luz del objetivo propuesto. Los resultados demuestran alta favorabilidad hacia la metodología de evaluación apoyada en Conectivismo debido a varios factores: la tecnología como lenguaje natural de los jóvenes de hoy, el océano de respuestas que Internet puede brindar, el acceso a recursos y respuestas y los elementos teóricos que constituyen las bases del Conectivismo como teoría de aprendizaje. Se concluye que, en un ambiente controlado y usando la evaluación como instrumento de aprendizaje, se puede alcanzar

This manuscript was submitted on May 15, 2020 and accepted for publication on September 06, 2020.

O. I. Trejos-Buriticá, Ingeniero de Sistemas, $\mathrm{PhD}$ en Ciencias de la Educación, Docente de Planta, Universidad Tecnológica de Pereira, Facultad de Ingenierías, Ingeniería de Sistemas y Computación (omartrejos@utp.edu.co). la motivación requerida para un aprendizaje efectivo con los jóvenes de hoy a partir de las bondades que proporciona la teoría del Conectivismo apoyándose en Aprendizaje Basado en el Cerebro.

Palabras Clave-Aprendizaje, Computación, Conectivismo, Evaluación, Programación

\section{INTRODUCCIÓN}

$\mathbf{Z}^{\mathrm{n}}$ un curso de programación de computadores dentro de un Eprograma de Ingeniería de Sistemas y Computación (o cualquiera de sus vertientes curriculares actuales) el concepto de evaluación es un elemento que se usa tradicionalmente para hacer una estimación del conocimiento adquirido de un estudiante y, a partir de los resultados, realizar una retroalimentación. La concepción teórica sobre la cual se ha realizado esta experiencia de investigación es que la evaluación puede constituirse, si así lo quiere el docente, en un instrumento de aprendizaje para el estudiante. Si se sabe que aprender a programar implica asimilar, apropiar y aplicar un conjunto de reglas que obedecen a una lógica diferente de la lógica deliberativa natural humana y que un curso de programación involucra métodos evaluativos que permitan saber hasta dónde dicha asimilación se ha dado, entonces los ingenieros docentes tienen ante sí un reto que debe dimensionarse apropiadamente.

La evaluación, en cualquier proceso de aprendizaje, merece una atención que va muy por encima del simple hecho del acto de calificar. Evaluar implica intentar definir el nivel en el cual el estudiante se encuentra en el proceso de asimilación de un nuevo conocimiento y poder establecer aquellas fronteras que el mismo estudiante tiene para que dicho proceso de asimilación sea posible y la manera de asimilarlas.

L. E. Muñoz-Guerrero Ingeniero de Sistemas, MSc en Ingeniería de Sistemas, Docente de Planta, Universidad Tecnológica de Pereira, Facultad de Ingenierías, Ingeniería de Sistemas y Computación (lemunozg@utp.edu.co).

G. R. Solarte-Martínez Ingeniero de Sistemas, MSc en Adminsitración Financiera, PhD en Informática, Docente Universidad Tecnológica de Pereira, Facultad de Ingenierías, Ingeniería de Sistemas y Computación (roberto@utp.edu.co). 
En el proyecto de investigación que inspira el presente artículo se busca establecer mecanismos, lo más objetivos posibles, que permitan hacer una comparación de la efectividad de dos metodologías. Las dos metodologías escogidas son: de una parte la evaluación por exámenes escritos de la manera como se ha hecho tradicionalmente en donde los estudiantes están aislados unos de otros trabajando de manera.

De otra parte, se ha optado por una evaluación escrita, con control de ventanas de tiempo, con acceso a Internet y posibilidad de discusión con compañeros de clase, en la cual el estudiante tiene opciones para fortalecer su aprendizaje (o para recordar lo olvidado) dado que la tensión propia de una evaluación parcial escrita genera algunas dificultades momentáneas con la información y el conocimiento que se encuentra en la memoria.

Este segundo camino para efectuar la evaluación implica un control riguroso de acceso a medios de comunicación por parte del docente, de resolución de las dudas presentadas y de consulta por los servicios que ofrece la Internet. Si bien es cierto que esto podría facilitar, in extremis, el camino para que los estudiantes obtengan una muy buena nota aún sin haberse preparado apropiadamente para su examen, será el docente quien tenga que diseñar una evaluación que sirva para el objetivo propuesto y que, además, capitalice las ventajas que ofrecen las nuevas tecnologías de la información y la comunicación a la luz de la teoría del Conectivismo estableciendo los controles apropiados.

El presente artículo contiene una experiencia novedosa pues combina y aprovecha elementos de la teoría de aprendizaje por descubrimiento, aprendizaje significativo, active Learning, aprendizaje basado en el cerebro y Conectivismo. Se justifica la experiencia desde la perspectiva de la innovación académica y didáctica pues vale la pena indagar estrategias que propendan por un aprendizaje tecnológico efectivo que, a diferencia de otras áreas de conocimiento, requieren la apropiación y aplicación de reglas lógicas diferentes a las naturales del ser humano.

Este es un producto derivado del proyecto de investigación "Desarrollo de un modelo metodológico para el aprendizaje de la programación imperativa en Ingeniería de Sistemas basado en aprendizaje significativo, aprendizaje por descubrimiento y un modelo de preferencias de pensamiento" de la Universidad Tecnológica de Pereira.

La temática planteada es importante para aquellos programas de ingeniería de sistemas cuyo perfil profesional está basado en el desarrollo de competencias en programación de computadores, así como en otros programas de formación profesional en donde la lógica de programación se tenga como un referente para el desarrollo del pensamiento lógico computacional así sea como asignatura complementaria. La investigación se realizó a lo largo de 8 semestres durante los años 2015, 2016, 2017 y 2018 en el programa Ingeniería de Sistemas y Computación de la Universidad Tecnológica de Pereira asignatura Programación II.

\section{Fundamentos TeÓRICOS}

El desarrollo de este artículo ha tenido unos pilares teóricos que, si bien no se pueden explicar plenamente como merecerían, por lo menos se pueden exponer brevemente por ser la base del corpus de esta investigación.

\section{A. Aprendizaje por Descubrimiento}

El aprendizaje por descubrimiento establece que todo lo que el ser humano descubre, dentro o fuera de un proceso de aprendizaje, queda almacenado en la memoria a largo plazo y podría aplicarlo en cualquier momento en situaciones similares o disímiles a aquellas en las cuales descubrió dicho nuevo conocimiento como en [1].

En el aprendizaje por descubrimiento la adquisición, apropiación y asimilación del conocimiento tiene tres procesos: el primero es la recepción, el segundo es la transformación y el tercero es la evaluación. En la recepción, el cerebro se vale de los sentidos para recibir nueva información y un significado que le brinde sentido a dicha información como en [2]. En este proceso es de gran importancia el conjunto de patrones, razonamientos y elementos de juicio previos que tiene el ser humano para asimilar el nuevo conocimiento y que, normalmente y en gran medida, son herencia del contexto extrainstitucional en el cual vive cotidianamente cada individuo.

La transformación corresponde a la búsqueda y hallazgo de sentido y significado del nuevo conocimiento dentro del diario vivir del estudiante y en el cual se intenta articular lo que se ha recibido como nuevo (a través de los sentidos) y las situaciones problémicas de la vida corriente en las cuales dicho conocimiento cobra cierto nivel de relevancia como se plantea en [2]. La evaluación es el proceso mediante el cual se establecen unos criterios que permitan revisar la transformación de la base cognitiva y la relación entre el nuevo conocimiento y las situaciones de la vida real donde podría ser aplicado, todo esto dentro de un proceso de descubrimiento que es el que se devela a la luz de la teoría de aprendizaje respectiva, tal como se presenta en [3].

Dentro de la teoría del aprendizaje por descubrimiento, la fascinación por lo insólito e innovador constituye un elemento de gran importancia pues genera la motivación necesaria para que el estudiante busque las respuestas que resuelven las preguntas que aquello insólito genera como en [4].

\section{B. Aprendizaje Significativo}

La teoría del aprendizaje significativo fundamenta su teoría en el hecho de que el ser humano aprende mucho más fácil si el nuevo conocimiento adquirido tiene significado y sentido como en [5]. Que tenga significado quiere decir que tiene aplicación en el mundo cotidiano que vive el estudiante, bien sea en lo vivencial o en lo académico, y que tenga sentido implica que pueda ser aplicado en diferentes contextos de forma que aporte para resolver algún tipo de problema o situación problémica. 
Según la teoría del aprendizaje significativo, lo más importante en un proceso de aprendizaje es lo que el estudiante ya sabe, razón por la cual esta teoría se fundamenta en la trilogía conformada por el conocimiento previo, el nuevo conocimiento y la actitud del estudiante. El conocimiento previo está conformado por el conjunto de juicios, modelos y teorías que subyacen al pensamiento del estudiante y que han sido adquiridos bien a lo largo de su vida académica o bien, y mayoritariamente, todas las situaciones vividas en el contexto extrainstitucional como en [6].

El nuevo conocimiento está conformado por todos aquellos modelos, teorías, estructuras o juicios que el estudiante aún no conoce o a los cuales no ha tenido acceso. Esto implica que cuando se habla de "nuevo" conocimiento se hace bajo la acepción de la RAE (Real Academia Española) según la cual nuevo es aquello "Distinto o diferente de lo que se había o se tenía aprendido".

La actitud del estudiante, como tercera componente del aprendizaje a la luz de esta teoría, se conforma de la motivación y de la capacidad que el estudiante desarrolle para establecer nexos entre el conocimiento previo y el nuevo conocimiento como se plantea en [7]. Esta capacidad se desarrolla a partir de la implementación de estrategias de aprendizaje dentro y fuera del aula que se convierten en un reto para el docente al momento de estudiarlas y aplicarlas en un proceso específico de aprendizaje. Por su parte la motivación está conformada por ánimos positivos y voluntad que el estudiante aporta para participar dentro del proceso de aprendizaje con el ánimo de aprender, es decir, apropiar, asimilar, aplicar y retroalimentar el conocimiento que se adquiere y que tiene una connotación de novedoso.

\section{Active Learning}

La estrategia de Active Learning es un camino a través del cual se puede lograr que el estudiante establezca, y se comprometa con, sus metas de aprendizaje, participe en el diseño del proceso para lograrlo y tenga elementos de juicio para determinar si dicho proceso ha sido exitoso como en [8].

Con esta estrategia el rol del docente cambia para convertirse en un acompañante guía y coequipero que proporciona indicaciones necesarias para que el estudiante, a la luz de sus propios objetivos, vaya ajustando su propio proceso de aprendizaje de manera que se involucre en el alcance de sus logros y sea partícipe de ellos.

\section{Brain Based Learning}

BBL describe una teoría de aprendizaje que se basa en las características fisiológicas propias del cerebro y que significan Brain Based Learning (Aprendizaje basado en el cerebro) según [9]. Según esta teoría es importante tener un conocimiento más detallado del cerebro para aplicar las teorías de aprendizaje de una forma tal que se articule con su fisiología. BBL se basa en un conjunto de principios que han sido producto de la investigación a nivel neurológico y que ha permitido cristalizar diferentes modelos del cerebro sin desconocer que el conocimiento detallado de este órgano es todavía incipiente en consideración con sus insondables capacidades como en [10].

Algunos de estos principios que han servido de fundamento para el desarrollo de este estudio se enuncian a continuación: a) el aprendizaje involucra la fisiología, b) el cerebro es social, c) la búsqueda de significado es innata, d) la búsqueda de significado ocurre por patrones, e) el cerebro procesa las partes y el todo simultáneamente, f) las emociones son críticas en la formación de patrones, g) el aprendizaje incluye atención enfocada y percepción periférica, h) el aprendizaje incluye procesos conscientes e inconscientes, i) en el cerebro contamos con la memoria espacial y la memoria rotante, $\mathrm{j}$ ) el aprendizaje se desarrolla, k) el aprendizaje complejo se mejora con los retos y se inhibe con los castigos, 1) cada cerebro está organizado de manera única como en [11].

\section{E. Teoría de Conectivismo}

El Conectivismo se puede describir como una teoría de aprendizaje para la era digital moderna propuesta por Stephen Downes y George Siemens. Según sus planteamientos, el aprendizaje se produce por medio de conexiones que se dan a partir de las diferentes redes que permiten la interacción entre seres humanos sobre una base de nodos y conexiones conceptuales que son las que definen el aprendizaje. El Conectivismo consagra como elementos constitutivos del aprendizaje conceptos como el caos, las redes, la complejidad y la auto-organización como en [12].

De acuerdo con el Conectivismo, el aprendizaje sucede dentro de entornos virtuales a partir de la interconexión entre elementos básicos que no están necesariamente bajo el control del aprendiz. Esta teoría acepta, por primera vez, que el aprendizaje puede residir fuera de nosotros mismos bien sea en una organización o en una base de datos como en [12]. El proceso de aprendizaje implica la conexión de conjuntos de información con cierto nivel de especialización y con unas conexiones que son las que nos permiten aprender de manera que mejoren un estado actual de conocimientos según lo que se presente en [13].

Bajo esta teoría, la interacción que se establecen entre los nodos de conocimiento se basa en la modificación veloz de las decisiones que se toman basado en ellos como en [13]. El pensamiento crítico emerge como una necesidad que permite distinguir entre información y conocimiento y que establece cuáles de ellos alteran el paisaje basado en determinadas decisiones. Algunos principios del Conectivismo son: a) aprendizaje y conocimiento se basan en opiniones diversas, $b$ ) el aprendizaje implica un proceso a través del cual se conectan nodos especializados que también pueden llamarse como fuentes de información, c) el aprendizaje puede llegar a residir en dispositivos no humanos, d) en tiempos modernos es muy importante tener una capacidad posible para saber más, e) el aprendizaje se facilita por conexiones que simplifiquen un proceso continuo, f) la toma de decisiones es un proceso de aprendizaje en su mismo accionar y en su misma definición que obliga a tener elementos de juicio acerca de qué se debe 
aprender y qué significado tiene ésta en los tres contextos de aprendizaje.

Según la teoría del Conectivismo, un conocimiento el día de hoy, es posible que esté desactualizado o inválido en tiempos futuros de manera que la revisión del conocimiento debe ser permanente pues incluso un mismo conocimiento y su relación con una situación específica puede cambiar tan solo con el paso del tiempo como se presenta en [14].

\section{F. Aprendizaje de la Programación}

La apropiación de los elementos que subyacen a la programación es de gran importancia en tiempos modernos dado que se han constituido en un camino que fundamenta y promueve el pensamiento computacional y el pensamiento crítico, base para el desarrollo profesional, la toma de decisiones y la articulación con la sociedad del mundo actual.

De allí que todo esfuerzo que se haga en relación con la búsqueda de caminos más expeditos para aprender a programar y que provengan de procesos investigativos, serán bienvenidos en el contexto académico pues no sólo aportan a la discusión sino que abren nuevas puertas para experimentar y lograr que dichos caminos se cristalicen y, por lo tanto, se logre el objetivo de que sean muchas las personas que aprendan a programar computadores no como una habilidad tecnológica sino como una competencia profesional según [15].

\section{MetodologíA}

Para el desarrollo de la investigación que inspira este artículo se trabajó con dos grupos en paralelo por cada semestre, uno con la asignatura Programación I (cuyo contenido versa sobre programación funcional) y otro con la asignatura Programación II (con contenido en programación imperativa), esto con el ánimo de que las estrategias y las temáticas no fueran a malograr los resultados del estudio.

Al principio del semestre se escogía, aleatoriamente, uno de los grupos para desarrollar la asignatura bajo la metodología tradicional de exposición magistral, planteamiento de ejercicios y resolución de problemas en clase, resolución de dudas, consulta dentro y fuera de la clase y evaluaciones parciales escritas individuales sin ningún tipo de ayuda adicional.

Con el otro grupo se adoptó la metodología que se expone en este artículo y que consistió en exposición magistral acompañada de desarrollo de ejercicios y resolución de problemas en clase, así como la resolución de dudas y consultas dentro y fuera de clase. En este grupo las evaluaciones parciales escritas se realizaron permitiendo que éstas fueran individuales pero cada estudiante podía tener a la mano algún tipo de dispositivo (celular, computador, Tablet, etc.) de forma que pudiera utilizarlo en una ventana de tiempo controlada por el docente y que accediera a los servicios de acceso y consulta que el mundo moderno provee.

El acceso a los dispositivos y ayudas adicionales no se permitió durante todo el tiempo destinado a las evaluaciones parciales.
En cada una de ellas se les informaba a los estudiantes a partir de qué momento podían acceder a los servicios de la web y usar sus dispositivos y el tiempo destinado para ello solamente estaba a discreción del docente investigador; también se les informaba por cuánto tiempo podían tener acceso a dichos servicios. Cabe acotar que los estudiantes, previo a las pruebas parciales escritas, no sabían que tenían esta facilidad de consulta durante la evaluación, pero si se les insistió en que llevaran dispositivos electrónicos con conectividad.

Es de anotar que este tiempo nunca fue superior a 3 minutos pues la idea es que aprovecharan el instante para acceder a las consultas, pero partiendo de lo que hubieren estudiado y no que terminaran copiándole a un compañero usando correo electrónico, whatsapp o algún otro servicio. En todo momento el proceso estuvo supervigilado por el docente. Se realizaron indicaciones acerca del tipo de consultas que podían hacer y se estableció que no se podían comunicar con otro estudiante para solicitarle que le enviara las respuestas puesto que no era este el objetivo de la estrategia lo cual se intentó controlar con vigilancia visual pero debe admitirse que pudo existir algún tipo de filtración por tal motivo el control más importante fue la toma de conciencia en la participación de la investigación a lo largo de todo el proceso de aprendizaje durante el semestre. Una vez se había terminado el tiempo de acceso y uso del dispositivo, se indicaba en dónde podían dejarlo de forma que, bajo la vigilancia del docente, se pudiera constatar que no se accedería a él por fuera de la ventana de tiempo permitida.

En cuanto a la aplicación del sustento teórico de la estrategia se aprovechó la teoría de aprendizaje significativo para plantear enunciados y situaciones problema que tuvieran conexión con las vivencias cotidianas de los estudiantes en su mundo extrainstitucional de forma que los ejercicios adquirieran sentido y significado. El aprendizaje por descubrimiento se aprovechó para motivar la resolución de los enunciados y para capitalizar la fascinación que provoca la tecnología dentro de una metodología que acude a las tecnologías modernas in situ.

La estrategia Active Learning ha servido, dentro de esta investigación, para motivar a que los estudiantes se empoderen de su propio proceso de aprendizaje, para que se preparen para las evaluaciones parciales como si no tuvieran ninguna otra opción de consulta durante dichas evaluaciones y para que sean conscientes de la importancia de aprender a largo plazo a partir de sus propios esfuerzos.

La teoría BBL se ha aprovechado para que los alumnos acepten opiniones diversas que enriquezcan sus consultas, para socializar el conocimiento a través de los medios electrónicos, para resolver el problema del significado de algunos conceptos en relación con la vida cotidiana, para provocar emociones y cuestionar los patrones de estudio establecidos, para aprovechar los aprendizajes conscientes, para concientizar al alumno acerca de metas alcanzables y castigos evitables y para que el cerebro trabaje tanto en la búsqueda de soluciones como en el aprovechamiento de las nuevas tecnologías de la información y la comunicación. 
La teoría del Conectivismo se ha utilizado para que el estudiante la conozca, la apropie, la utilice y la aproveche como base de su avance en el conocimiento dentro del proceso de aprendizaje y alcance de los logros establecidos en el curso de programación utilizando dispositivos electrónicos que posibiliten fortalecer el aprendizaje durante la presentación de las pruebas parciales escritas.

La programación de computadores ha sido la temática central que ha permitido el corpus sobre el cual se han aplicado tanto las teorías que giran alrededor de esta investigación como las estrategias de aprendizaje con las cuales se espera se mejore el camino para simplificar el logro de los objetivos de aprendizaje establecidos.

De esta forma, se ha presentado la evaluación como una herramienta de aprendizaje de manera que sea motivante y no restrictiva con cada grupo al tiempo que se ha monitoreado lo que sucede con el grupo en su conjunto. La Tabla I presenta las estrategias y los tiempos que se estimaron apropiados para el desarrollo de esta investigación.

TABLA I.

ESTRATEGIAS Y TIEMPOS ESTIMADOS

\begin{tabular}{|c|c|c|c|}
\hline Estrat & $\begin{array}{l}\text { Tiempo } \\
\text { Estim. } \\
\text { (en min) }\end{array}$ & $\begin{array}{c}\text { Frec } \\
\text { (en una } \\
\text { misma } \\
\text { Eval) }\end{array}$ & $\begin{array}{l}\text { Soporte } \\
\text { Teórico }\end{array}$ \\
\hline $\begin{array}{l}\text { Consulta por } \\
\text { NTIC }\end{array}$ & 3 & 2 & $\begin{array}{l}\text { Ap. Signif. } \\
\text { Ap. x Descub. }\end{array}$ \\
\hline $\begin{array}{l}\text { Copartir con } \\
\text { compañero }\end{array}$ & 2 & 1 & $\begin{array}{l}\text { Brain Based Learning } \\
\text { Active Learning }\end{array}$ \\
\hline $\begin{array}{l}\text { Preguntar al } \\
\text { docente }\end{array}$ & 10 & 2 & $\begin{array}{c}\text { Ap. Signif. } \\
\text { Active Learning }\end{array}$ \\
\hline $\begin{array}{l}\text { Discutir con } \\
\text { Compañero }\end{array}$ & 3 & 2 & $\begin{array}{c}\text { Ap. X Descub. } \\
\text { Brain Based Learning }\end{array}$ \\
\hline
\end{tabular}

Al final de semestre se realizó a los estudiantes (de ambos grupos) un examen final que incluyera todos los temas vistos durante el semestre. Este examen final se diseñó de forma que fuera evaluativo y cuya presentación obedecía a los parámetros convencionales (individual, desconectado y sin preguntas). La Tabla II presenta un resumen de la cantidad de estudiantes involucrados en la investigación que inspira este artículo.

TABLA II.

ESTUDIANTES INVOLUCRADOS

\begin{tabular}{cccc}
\hline \hline Año & Sem & Cant & M C* \\
\hline \multirow{2}{*}{2015} & I & 24 & \\
& II & 24 & $*$ \\
2016 & I & 43 & $*$ \\
& II & 41 & \\
2017 & I & 42 & $*$ \\
& II & 44 & \\
2018 & I & 46 & $*$ \\
T o t a 1 & 42 & $*$ \\
\hline \hline \multicolumn{4}{c}{ Fuente: Elaboración propia } \\
M C $=$ Grupo metodología con Conectivismo
\end{tabular}

Durante todo el proceso de investigación se procuró que los grupos tuvieran un número equivalente un número de estudiantes.

\section{Resultados}

Las Tablas IIIa y IIIb presentan el promedio de los resultados cuantitativos obtenidos en las evaluaciones parciales (P1, P2 y P3) tanto del subgrupo con el cual se trabajó la metodología tradicional como del subgrupo en donde la metodología se basó en Conectivismo.

TABLA IIIA

Resultados Notas Parciales MT

\begin{tabular}{|c|c|c|c|c|c|}
\hline \multirow{2}{*}{ Año } & \multirow{2}{*}{ Sem } & \multicolumn{4}{|c|}{ Metodología Tradicional } \\
\hline & & $\mathrm{P} 1$ & $\mathrm{P} 2$ & P3 & Prom \\
\hline \multirow{2}{*}{2015} & I & 3,4 & 4,0 & 3,9 & 3,8 \\
\hline & II & 3,3 & 4,1 & 3,5 & 3,6 \\
\hline \multirow{2}{*}{2016} & I & 3,5 & 3,9 & 3,8 & 3,7 \\
\hline & II & 3,6 & 3,8 & 3,7 & 3,7 \\
\hline \multirow{2}{*}{2017} & I & 3,9 & 3,5 & 3,6 & 3,7 \\
\hline & II & 3,2 & 3,2 & 3,5 & 3,3 \\
\hline \multirow{2}{*}{2018} & I & 3,2 & 3,0 & 3,4 & 3,2 \\
\hline & II & 3,1 & 3,2 & 3,0 & 3,1 \\
\hline \multicolumn{2}{|c|}{ Promedios } & 3,4 & 3,6 & 3,6 & 3,5 \\
\hline
\end{tabular}

Fuente: Elaboración propia

* MT = metodología tradicional

TABLA IIIB.

RESUlTAdOS NOTAS PARCIALES MC

\begin{tabular}{|c|c|c|c|c|c|}
\hline \multirow[t]{2}{*}{ Año } & \multirow[t]{2}{*}{ Sem } & \multicolumn{4}{|c|}{$\begin{array}{l}\text { Metodología } \\
\text { Conectivismo }\end{array}$} \\
\hline & & P1 & $\mathrm{P} 2$ & P3 & Prom \\
\hline \multirow{2}{*}{2015} & I & 4,3 & 4,2 & 4,6 & 4,4 \\
\hline & II & 4,5 & 4,5 & 4,7 & 4,6 \\
\hline \multirow{2}{*}{2016} & I & 4,2 & 4,3 & 4,5 & 4,3 \\
\hline & II & 4,7 & 4,6 & 4,6 & 4,6 \\
\hline \multirow{2}{*}{2017} & I & 4,5 & 4,3 & 4,4 & 4,4 \\
\hline & II & 4,5 & 4,4 & 4,6 & 4,5 \\
\hline \multirow{2}{*}{2018} & $\mathrm{I}$ & 4,6 & 4,4 & 4,5 & 4,4 \\
\hline & II & 4,3 & 4,6 & 4,4 & 4,6 \\
\hline \multicolumn{2}{|c|}{ Promedios } & 4,5 & 4,4 & 4,5 & 4,5 \\
\hline
\end{tabular}

La Tabla IV expone el promedio de los resultados obtenidos en el examen final que correspondió a una prueba similar para ambas metodologías.

TABLA IV.

Resultados Examen Final

\begin{tabular}{|c|c|c|c|c|}
\hline Año & Sem & MetTrad & MetCon & Difer \\
\hline \multirow{2}{*}{2015} & I & 3,8 & 4,7 & 0,9 \\
\hline & II & 3,6 & 4,8 & 1,2 \\
\hline \multirow{2}{*}{2016} & I & 3,5 & 4,7 & 1,2 \\
\hline & II & 3,7 & 4,7 & 1,0 \\
\hline \multirow{2}{*}{2017} & I & 3,5 & 4,6 & 1,1 \\
\hline & II & 3,4 & 4,7 & 1,3 \\
\hline \multirow{2}{*}{2018} & I & 3,2 & 4,8 & 1,6 \\
\hline & II & 3,3 & 4,6 & 1,3 \\
\hline \multicolumn{2}{|c|}{ Promedios } & 3,5 & 4,7 & 1,2 \\
\hline
\end{tabular}


La observación directa por parte del docente sobre el avance de cada estudiante en relación con la apropiación del conocimiento de la programación de computadores, al margen de lo puramente cuantitativo, se refleja en la Tabla $\mathrm{V}$ en donde se consigna el concepto cualitativo de si cada estudiante aprendió a programar o no y a cuál grupo pertenecía.

TABLA V.

Resultados Cualitativos

\begin{tabular}{|c|c|c|c|c|c|}
\hline \multirow{3}{*}{ Año } & \multirow{3}{*}{ Sem } & \multicolumn{4}{|c|}{$\begin{array}{l}\text { ¿Aprendió a programar? } \\
\text { (concepto docente) }\end{array}$} \\
\hline & & \multicolumn{2}{|c|}{ M T } & \multicolumn{2}{|c|}{$\mathrm{MC}$} \\
\hline & & $\mathrm{Si}$ & No & $\mathrm{Si}$ & No \\
\hline \multirow{2}{*}{2015} & $\mathrm{I}$ & 12 & 9 & & \\
\hline & II & & & 17 & 5 \\
\hline \multirow{2}{*}{2016} & $\mathrm{I}$ & & & 19 & 4 \\
\hline & II & 14 & 7 & & \\
\hline \multirow{2}{*}{2017} & I & & & 20 & 2 \\
\hline & II & 13 & 11 & & \\
\hline \multirow{2}{*}{2018} & I & 21 & 5 & & \\
\hline & II & & & 19 & 3 \\
\hline
\end{tabular}

\section{DISCUSIÓN}

La metodología adoptada por grupos tiene la gran ventaja de poder disponer de los estudiantes para aquellas pruebas que se considere necesaria realizar simultánea e igualmente posibilita el desarrollo de todas las comparaciones y cruces de variables posibles pues depende del docente investigador que está a cargo de la asignatura.

Si el objetivo de cada asignatura consiste en que los estudiantes hagan suyas las reglas de la lógica de programación a la luz de sendos paradigmas, entonces una metodología como la que se sugiere ayuda a que, por la vía de la socialización entre los alumnos, esto sea posible.

Los resultados presentados, desde de diferentes estilos de enseñanza, pero bajo los mismos objetivos, podrían darnos elementos de juicio para facilitar una generalización incluso en asignaturas de otras áreas. Sin embargo, lo obtenido da un claro indicio de que es posible aprovechar las evaluaciones escritas como mecanismos para alcanzar los objetivos de aprendizaje a la luz de características propias del cerebro y dentro del marco del lenguaje natural de los jóvenes de hoy: las nuevas tecnologías de la información y la comunicación.

La adopción de estrategias innovadoras durante una evaluación parcial es posiblemente uno de los retos más interesantes que tiene un docente en tiempos modernos. El hecho de saber que dentro de una evaluación parcial los estudiantes viven, por razones naturales, una tensión inherente al proceso evaluativo (en los términos como se concibe tradicionalmente) proporciona caminos para que el cerebro del estudiante, dentro del marco de sus principios rectores, pueda "aprender" durante la evaluación.

Esta misma tensión podría convertirse en un factor favorable durante las pruebas escritas si se tiene en cuenta que las emociones son determinantes en un proceso de aprendizaje y es por ello que autorizar, de manera repentina, estrategias que los estudiantes no esperan, logra una apropiación de conocimiento más inmediata y efectiva que la que podría lograrse por caminos tradicionales en pro del aprendizaje siempre que se cuente con ventanas de tiempo y ambientes tecnológicos controlados. Los resultados obtenidos parecieran indicar que así fue.

Pensar en que por unos minutos los estudiantes puedan consultar al compañero, al profesor o a un medio electrónico significa derribar paradigmas que hasta ahora han sido parte de lo puramente tradicional y que no necesariamente logran que la evaluación facilite el aprendizaje, aunque si permitan obtener notas cuantitativas de las pruebas realizadas por los caminos conocidos. El examen final se ha realizado en los términos tradicionales en que se presenta una prueba escrita solo para tener una forma de comparar los resultados obtenidos con estrategias innovadoras y su efecto dentro de los procesos que se han utilizado normalmente en el aula.

Una extensión posible a este estudio implicaría realizar la evaluación final en los términos innovadores que involucra esta investigación, es decir, aplicando Brain Based Learning y Conectivismo. Allí sería posible cotejar resultados, tanto con métodos convencionales como con métodos innovadores, y verificar en lo cualitativo y en lo cuantitativo hasta donde mejora el aprendizaje de la programación, que finalmente es el gran objetivo de la asignatura.

En las Tablas III, como era de esperarse, los resultados cuantitativos de la metodología propuesta en esta investigación siempre son, uno a uno, mejores que los resultados obtenidos con el grupo que se condujo por los mecanismos tradicionales. Llama la atención la alta diferencia entre unos y otros. Sin embargo la Tabla IV, que resume los resultados obtenidos en la prueba final de cada curso, se confirma lo que parecieran decir los datos en las pruebas parciales. Se observa que los resultados cuantitativos son notoriamente mejores con los estudiantes en quienes se aplicó la metodología objeto de esta investigación que en los estudiantes que se atendieron por el método tradicional de "evaluación".

Finalmente, en la Tabla V se presenta la opinión resumida del docente en relación con lo que era el objetivo inicial de la asignatura que consiste en el aprendizaje de la programación de computadores y cuya opinión se basa en la observación del progreso del estudiante. Si bien podría pensarse en que esta opinión pudiera estar impregnada de algo de subjetividad, se ha tratado de ser lo más objetivo posible dado que lo que se pretende es que estos resultados, y su discusión asociada, enriquezcan el debate al respecto de las estrategias y metodologías efectivas en el aula para que los alumnos aprendan a programar.

De acuerdo con lo observado en la Tabla V, la efectividad de la metodología es de un $85 \%$ con los estudiantes con metodología basada en BBL y Conectivismo frente a un 60\% de efectividad con los estudiantes que se atendieron con metodología tradicional. A partir de la experiencia, de los resultados obtenidos y de las observaciones realizadas en esta discusión, 
es posible lograr que se incremente la efectividad de la metodología propuesta (aproximándose a un $90 \%$ lo cual sería un muy buen indicador).

También estos resultados indican que la metodología tradicional todavía tiene algunos elementos que aportan aprendizaje, sin embargo, la comodidad de los estudiantes en una metodología como la que inspira este artículo de investigación es notoriamente favorable al proceso de aprendizaje y eso la ubica en una instancia superior a la tradicional.

\section{CONCLUSIONES}

Aprovechando y aplicando la teoría de aprendizaje basado en el cerebro (Brain Based Learning) y las facilidades que el Conectivismo (como teoría y práctica) provee a los jóvenes de hoy, pareciera ser claro que el aprendizaje se mejora si se incorporan estrategias que tengan en cuenta estas teorías y su aplicación en experiencias investigativas como la que inspira este artículo.

Se requiere que los docentes ingenieros se preparen no solo en la profundización de su conocimiento disciplinar (lo cual es absolutamente necesario) sino que acudan a teorías, metodologías, modelos y estrategias que posibiliten alcanzar, con sus respectivos grupos, los objetivos que se hayan propuesto tanto en lo teórico como en lo práctico en relación con la programación de computadores.

Se constituye en un reto muy interesante y provocador la incorporación de nuevas estrategias que dinamicen las evaluaciones de las asignaturas conducidas por Ingenieros y que propendan por el mejoramiento del aprendizaje que incluye apropiación, asimilación, aplicación, retroalimentación y evaluación de nuevos conocimientos adquiridos, así como el cuestionamiento respectivo de conocimientos previos que, por este camino, pueden actualizarse, mejorarse o descartarse.

Finalmente, resulta de gran utilidad que los ingenieros docentes puedan concebir la evaluación como una herramienta efectiva de aprendizaje si la articulan con las teorías y las estrategias apropiadas, tal como se realizó en esta experiencia investigativa.

\section{REFERENCIAS}

[1] J. Brune "Actos de Significado". Madrid - España: Alianza Editorial. Año 2009.

[2] J. Bruner, "Hacia una teoría de la instrucción". México: Editorial Limusa. Año 2006.

[3] F. Diaz Barriga, , \& G. Hernandez Rojas, "Estrategias docentes para un aprendizaje significativo". México: McGraw Hill. Año 2002.

[4] O. Trejos Buriticá, "Significado y Competencias". Pereira (Risaralda) - Colombia: Editorial Papiro. Año 2012

[5] D. Ausubel "The Acquisition and Retention of Knowledge." Washington - USA: Springer. Año 2012.

[6] A. Woolfolk, "Psicología Educativa." México: Prentice Hall. Año 1999.

[7] L. Serrano Cámara., M. Paredes Velasco,., \& et al. An "evaluation of students motivation in computer supported collaborative learning of programming concepts". Computers in Human Behavior(31), 499 - 508. Año 2014. https://doi.org/10.1016/j.chb.2013.04.030

[8] M. Prince. Does "Active Learning work? Journal of Engineering Education", 93(3), 223-231 ., Julio de 2004. DOI: 10.1002/j.21689830.2004.tb00809.x

[9] W. Herrmann "The whole brain bussiness book. New York": McGraw Hill. Año 2015.

[10] A. Ballester Valori, "Meaningful Learning in practice. Islas Canarias": Universitat de les Illes Ballears Año 2011.

[11] A. Davis. "The credentials of brain based learning. Journal of Philosophy of Education", 38(1), 21. Año 20014. DOI: 10.1111/j.0309-8249.2004.00361

[12] E Jensen,.. "The learning brain. NY": Brain Store Inc. Año 1994

[13] H. Smidt,, M. Thornton,, \& A. Kaveh , "The future of social learning": a novel approach to connectivism. Proceedings of the 50th Hawaii International Conference on System Sciences , 2116 2125. Año 2017. DOI: 10.24251/HICSS.2017.256

[14] J. Johannsson, \& F. Elgh, "Applying Connectivism to EngineeringKnowledge to Support the AutomatedBusiness." In: 24th ISPE International Conference on Transdisciplinary Engineering (TE2017), 621 - 628. Año 2017. DOI: 10.3233/978-161499-779-5-621

[15] O. Trejos Buriticá . "Lógica de Programación.” Bogotá: Ediciones de la U. Año 2017

[16] R. Harlterman,. "Learning to program with Python." Washington, USA: Richard Halterman Edit. Año 2011

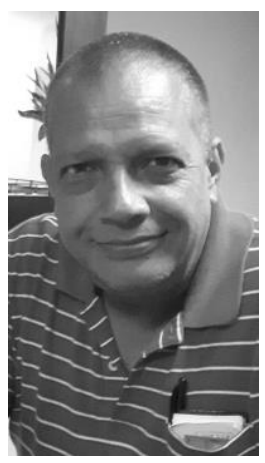

Omar Iván Trejos Buriticá, Ingeniero de Sistemas. Especialista en Instrumentación Física. MSc en Comunicación Educativa. PhD en Ciencias de la Educación. Docente de planta categoría Titular, Programa Ingeniería de Sistemas y Computación, Facultad de Ingenierías, Universidad Tecnológica de Pereira. Autor de varios libros de programación y de una buena cantidad de artículos de investigación científica educativa en el área de la programación de computadores sobre aproximación a la optimización de procesos de enseñanza y aprendizaje dentro del contexto de la formación de ingenieros con perfil tecnológico. ORCID: https://orcid.org/0000-0002-3751-6014.

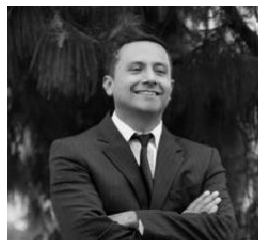

Luis Eduardo Muñoz Guerrero. Ingeniero de Sistemas. MSc en Ingeniería de Sistemas. Docente Titular de Planta Universidad Tecnológica de Pereira, con 15 años de experiencia en el campo de la formación universitaria. Autor de libros académicos y de investigación. Ha publicado artículos en revistas especializadas nacionales e internacionales. Su área de Investigación se centra en los procesos de enseñanza y aprendizaje de la Programación. ORCID: http://orcid.org/00000002-9414-6187. 


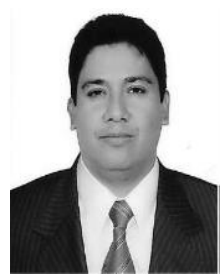

Guillermo Roberto Solarte Martínez, Doctor en Informática de la Universidad Pontificia de Salamanca con sede Madrid España Suficiencia investigativa, D.E.A Universidad Pontificia de Salamanca con sede Madrid España, Magister en Investigación de Operativa y Estadística de la Universidad Tecnológica de Pereira. Ingeniero de Sistemas de la Universidad Cooperativa de Colombia. Profesor ISC-UTP, Transitorio Titular en la Facultad de Ingenierías. Ha publicado artículos en revistas nacionales especializadas. Libros publicados: Guía didáctica de estructuras de datos, Pertenece a los grupos de investigación Grupo Grande Grupo de Avanzada en Desarrollo de Software, Grupo GIA - Grupo de investigación en Inteligencia Artificial, Semillero de Investigación GNTO Grupo de Nuevas técnicas de búsqueda y de optimización. ORCID: https://orcid.org/0000-0001-5147-7798 